\title{
UM PERFIL DINÂMICO DE PLUMAS ATRAVÉS DA ANÁLISE PERTURBATIVA DA EQUAÇÃO DA DISPERSÃO DE POLUENTES EM COORDENADAS CILÍNDRICAS
}

\author{
Cláudio C. Pellegrini ${ }^{1}$, Bardo E. J. Bodmann ${ }^{2}$ \\ ${ }^{1}$ UFSJ, São João del-Rei, MG; ${ }^{2}$ UFRGS, Porto Alegre, RS
}

\section{RESUMO}

O presente trabalho usa métodos de perturbação e coordenadas cilíndricas para simplificar a equação advectivo-difusiva da dispersão de poluentes. Os resultados corroboram os de PELLEGRINI et al. (2013), obtidos em coordenadas cartesianas, e exibem interessantes simplificações resultantes da simetria axial inerente ao problema.

\section{SUMMARY}

This study uses perturbation techniques and cylindrical coordinates to simplify the advective-diffusive pollutant dispersal equation. The results corroborate the ones obtained by PELLEGRINI et al. (2013) in Cartesian coordinates and show some interesting simplifications due to the inherent axial symmetry of the problem.

\section{1- INTRODUÇÃO}

Em um trabalho recente, PELLEGRINI et al. (2013) utilizaram métodos de perturbação para simplificar a equação 3D advectivo-difusiva da dispersão de poluentes à partir de uma fonte localizada. A revisão lá apresentada mostra que a maioria dos trabalhos anteriores usa coordenadas cartesianas ortogonais, a despeito da geometria da pluma ser mais bem descrita em coordenadas cilíndricas. O presente trabalho refaz a análise de PELLEGRINI et al. (2013) em coordenadas cilíndricas mostrando que os resultados lá obtidos também aqui o são, mas com a vantagem de o problema ser inerentemente bidimensional neste sistema.

\section{2- ANÁLISE MATEMÁTICA}

Aplicando a decomposição de Reynolds às eqs. da conservação da massa (ECM) de ar e de poluente, para o escoamento de uma pluma $3 \mathrm{D}$, transiente, incompressível, em coordenadas cilíndricas, sem produção de poluente por reação química, com simetria axial e tomando a média temporal segue-se

$$
\begin{aligned}
& \frac{1}{r} \frac{\partial\left(r \overline{v_{r}}\right)}{\partial r}+\frac{\partial \bar{v}_{z}}{\partial z}=0, \quad \frac{1}{r} \frac{\partial\left(r v_{r}^{\prime}\right)}{\partial r}+\frac{\partial v_{z}{ }^{\prime}}{\partial z}=0 \\
& \frac{\partial \bar{c}}{\partial t}+\bar{v}_{r} \frac{\partial \bar{c}}{\partial r}+\bar{v}_{z} \frac{\partial \bar{c}}{\partial z}=D\left[\frac{1}{r} \frac{\partial}{\partial r}\left(r \frac{\partial \bar{c}}{\partial r}\right)\right.+\frac{\left.\partial^{2} \bar{c}\right]}{\left.\partial z^{2}\right]} \\
&-\left[\frac{\partial \overline{\left(c^{\prime} v_{r}^{\prime}\right)}}{\partial r}+\frac{\overline{c^{\prime} v_{r}^{\prime}}}{r}+\frac{\partial\left(\overline{c^{\prime} v_{z}}\right)}{\partial z}\right]
\end{aligned}
$$

em $0 \leq r \leq R_{p}, 0 \leq \theta \leq 2 \pi, 0 \leq z \leq L$, sendo $L$ o comprimento da pluma, $R_{p}$ seu raio e $L \gg R_{p}$. Aqui, $c$ é a concentração volumétrica do poluente, $\boldsymbol{V}=\left(v_{r}, v_{\theta}, v_{z}\right)$ é o vetor velocidade e $D$ é a difusividade (molecular) binária do poluente no ar. As condições de fronteira 
são $\quad \bar{v}_{r}\left(R_{p}, \theta, z, t\right)=\bar{v}_{r 0}, v_{z}(r, \theta, L, t) \rightarrow v_{z 0} \quad, \quad \bar{c}\left(R_{p}, z, t\right)=0 \quad, \quad \partial \bar{c} / \partial r(0, z, t)=0$, $\bar{c}(r, 0, t)=c_{s}(t), \bar{c}(r, L, t) \rightarrow 0$, em que $c_{s}(t)$ é a concen-tração na fonte e $\boldsymbol{V}_{\mathbf{0}}=\left(v_{r 0}, v_{z 0}\right)$ é o campo de velocidade não perturbado pela pluma.

Para adimensionalizar as eqs. anteriores propomos $V_{r}=\bar{v}_{r} / V_{c}, V_{z}=\bar{v}_{z} / U_{g}$, em que $V_{c}$ é uma velocidade característica, arbitrária para o presente caso, $T=t / t_{c}$, sendo $t_{c}$ um tempo característico das variações da fonte emissora, $R_{1}=r / L, Z=z / L$, para o ar, sendo $L$ o comprimento horizontal característico da CLA, $R_{2}=r / R_{p}(z), Z=z / L$, para o poluente $\left(\operatorname{logo} R_{2}=R_{1} L / R_{p}\right), C=\bar{c} / c_{s}(t), V_{r}{ }^{\prime}=v_{r}^{\prime} / u_{*}, V_{z}{ }^{\prime}=v_{z}{ }^{\prime} / u_{*}, C^{\prime}=c^{\prime} / c_{*}$, em que $c_{*}=-\overline{c^{\prime} v_{r}^{\prime}} / u_{*}$. Para estirar as coordenadas radiais usamos $\tilde{R}_{1}=R_{1} / \varepsilon$ e $\tilde{R}_{1}=\left(1-R_{1}\right) / \varepsilon$, nas ECM do ar e do poluente, respectivamente, com $\varepsilon$ variando em ]0,1] e $0 \leq R_{1} \leq 1$, pois a física da CLA indica que para o ar a camada limite está localizada próximo à superfície e BUSH (1992) sugere que para eqs. como a ECM de poluente as camadas limite localizam-se nos extremos do domínio $\left(r=R_{p}\right)$. Obtém-se para a ECM do ar que $\partial V_{r} /\left(\varepsilon \partial \widetilde{R}_{1}\right)=$ $O\left(\partial V_{z} / \partial Z\right)$ qualquer que seja $O\left(\varepsilon \tilde{R}_{1}\right)$ e a ECM de poluente fica

$$
\begin{aligned}
\varepsilon_{t} \frac{\partial C}{\partial T}+\left[-V_{r} \frac{\partial C}{\partial \tilde{R}_{1}}+V_{z} \frac{\partial C}{\partial Z}\right] \\
=\varepsilon_{D}\left[\frac{\varepsilon^{-2}}{\left(1-\varepsilon \tilde{R}_{1}\right)} \frac{\partial}{\partial \tilde{R}_{1}}\left(\left(1-\varepsilon \tilde{R}_{1}\right) \frac{\partial C}{\partial \tilde{R}_{1}}\right)+\frac{\partial^{2} C}{\partial Z^{2}}\right] \\
-\varepsilon_{*} \varepsilon_{C *}\left[-\frac{\partial\left(C^{\prime} V_{r}^{\prime}\right)}{\varepsilon \partial \tilde{R}_{1}}+\frac{C^{\prime} V_{r}^{\prime}}{1-\varepsilon \tilde{R}_{1}}+\frac{\partial\left(C^{\prime} V_{z}^{\prime}\right)}{\partial Z}\right]
\end{aligned}
$$

em que os parâmetros pequenos são $\varepsilon_{t}=L / U_{g} t_{c}, \varepsilon_{L}=R_{p} / L, \varepsilon_{D}=D / U_{g} L=1 / \operatorname{Re}_{D}, \varepsilon_{*}=$ $u_{*} / U_{g}$ e. $\varepsilon_{c *}=c_{*} / c_{s}$. Valores típicos para as variáveis envolvidas em atmosfera neutra podem ser obtidos em PELLEGRINI et al. (2013) e $t_{c}=3.600 \mathrm{~s}$. Segue-se que $\varepsilon_{D} \ll\left(\varepsilon_{*} \varepsilon_{c *}\right)^{2}$. Simbolicamente a eq. anterior fica

$$
\begin{aligned}
& \left.O\left(\varepsilon_{t}\right)+O(1)=\varepsilon_{D}\left[O\left(\frac{1}{\varepsilon^{2}}\right)+O(1)\right]+\varepsilon_{*} \varepsilon_{c *}\left[O\left(\frac{1}{\varepsilon}\right)+O(1)\right)\right] \\
& \text { Ac Adv } \quad \operatorname{Dif}_{\mathrm{r}} \quad \operatorname{Dif}_{\mathrm{z}} \quad \mathrm{T}_{\mathrm{r} 1} \quad \mathrm{~T}_{\mathrm{r} 2 \mathrm{e}} \mathrm{T}_{\mathrm{z}}
\end{aligned}
$$

Os limites distintivos são mostrados na Tabela 1 , que usa $\varepsilon_{D} \ll\left(\varepsilon_{*} \varepsilon_{c *}\right)^{2}$. Resultam apenas dois limites distintivos: $\varepsilon=\varepsilon_{*} \varepsilon_{c *}$ e $\varepsilon=\varepsilon_{D} / \varepsilon_{*} \varepsilon_{c *}$ pois não há região em que os ter-

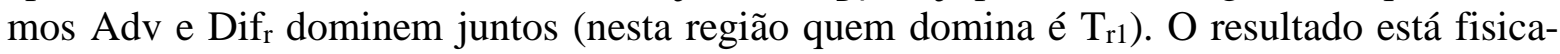
mente representado na Fig. 1.

\section{3- CONCLUSÕES}

O presente trabalho analisou o perfil de concentração em plumas de poluentes resultantes da equação da advecção-difusão utilizando a própria pluma e um sistema de coordenadas cilíndrico associado. A análise mostrou que a pluma pode ser dividida em regiões onde predominam em primeira ordem de aproximação determinados termos da equação original em coordenadas cilíndricas. Os resultados corroboram as conclusões anteriores de Pellegrini et al. (2013) no que diz respeito ao efeito físico predominante em cada região. 
Tabela 1: Limites distintivos da ECM de poluente

\begin{tabular}{|c|c|c|c|c|c|}
\hline \multirow{3}{*}{$\begin{array}{l}\text { Termos de } \\
\text { mesma ordem }\end{array}$} & \multirow{3}{*}{$\begin{array}{l}\text { Limite } \\
\text { distintivo }\end{array}$} & \multicolumn{3}{|c|}{$\begin{array}{l}\text { Ordem de grandeza dos termos da } \\
\text { equação }\end{array}$} & \multirow{3}{*}{$\begin{array}{l}\text { Termos } \\
\text { domi- } \\
\text { nantes }\end{array}$} \\
\hline & & Adv & $\operatorname{Dif}_{r}$ & $\mathrm{~T}_{\mathrm{r} 1}$ & \\
\hline & & 1 & $\varepsilon_{D} / \varepsilon^{2}$ & $\varepsilon_{*} \varepsilon_{C *} / \varepsilon$ & \\
\hline Adv e $\operatorname{Dif}_{r}$ & $\varepsilon=\sqrt{\varepsilon_{D}}$ & 1 & 1 & $\frac{\varepsilon_{*} \varepsilon_{c *}}{\sqrt{\varepsilon_{D}}}$ & $\mathrm{~T}_{\mathrm{r} 1}$ \\
\hline$A d v$ e $T_{r 1}$ & $\varepsilon=\varepsilon_{*} \varepsilon_{C *}$ & 1 & $\frac{\varepsilon_{D}}{\left(\varepsilon_{*} \varepsilon_{C *}\right)^{2}}$ & 1 & Adv e $T_{r 1}$ \\
\hline $\operatorname{Dif}_{\mathrm{r}}$ e $T_{\mathrm{r} 1}$ & $\varepsilon=\frac{\varepsilon_{D}}{\varepsilon_{*} \varepsilon_{C *}}$ & 1 & $\frac{\left(\varepsilon_{*} \varepsilon_{C *}\right)^{2}}{\varepsilon_{D}}$ & $\frac{\left(\varepsilon_{*} \varepsilon_{C *}\right)^{2}}{\varepsilon_{D}}$ & $\operatorname{Dif}_{\mathrm{r}}$ e $T_{\mathrm{r} 1}$ \\
\hline
\end{tabular}

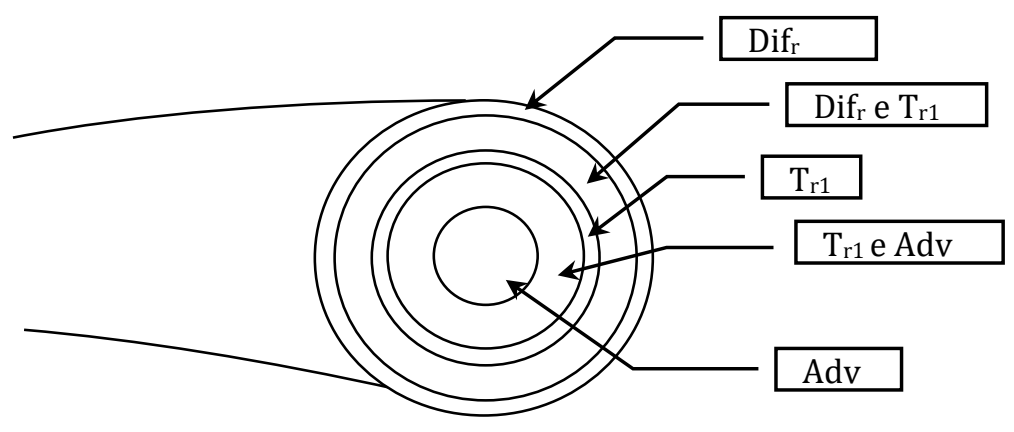

Fig. 1. Regiões de dominância dos termos na ECM poluente.

Agradecimentos: à FAPEMIG e à CAPES.

\section{4- REFERÊNCIAS BIBLIOGRÁFICAS}

BUSH, A. W. Perturbation Methods for Engineers and Scientists, CRC Press, USA, 320 pp. 1992

PELLEGRINI, C. C. ; BUSKE, D. ; BODMANN, B. E. J. ; VILHENA, M. T. A First Order Pertubative Analysis of the Advection-Diffusion Equation for Pollutant Dispersion in the Atmospheric Boundary Layer, Am. J. Environ. Eng., v. 3, p. 48-55, 2013 